\title{
Self-growing Colored Petri Net for offshore wind turbines maintenance systems
}

\author{
Pérez M. ${ }^{1}$, Correcher A. ${ }^{1}$, García E. ${ }^{1}$, Morant F. ${ }^{1}$ and Quiles E. ${ }^{1}$ \\ ${ }^{1}$ Department of Fault Diagnosis, Industrial Automation Institute (AI2) \\ E.T.S.I.I., Universidad Politécnica Valencia \\ 46022 Valencia (Spain) \\ Phone: (+34) 963877000 ext 88448, e-mail: mjopecol@upv.es, ancorsal@upvnet.upv.es, egarciam@isa.upv.es, \\ fmorant@isa.upv.es, equiles@isa.upv.es
}

\begin{abstract}
.
The offshore wind turbines have been developed in a lot of aspects in the last years, but the big companies are still researching for new techniques that help improve the systems.

We propose a new methodology to implement the automatic maintenance system using self-growing colored Petri nets developed in Labview, extendable to other industry systems.
\end{abstract}

\section{Key words}

Wind power turbine, Self-growing Colored Petri Net, offshore, optimal maintenance, cost.

\section{Introduction}

In this article, an optimal management system for offshore wind turbines is presented. It has been developed based in Colored Petri Nets (CPN) implemented in Labview.

The system we present in an automatic maintenance system created with Colored Petri Nets and the subsystem that compose it, but also with the new methodology and libraries developed to fulfil the project requirements.

The objective of the system is to work automatically joining the diverse subsystems in order group the maintenance order and select the optimal solution, analyzing costs, shipment and other factors. The orders must be revised and accepted by authorized personal.

The main characteristic of the system is the possibility of work with any number of orders, and create and erase them during the execution of the program without affecting the system. This is possible due to the coordinated job of Labview and Excel.

\section{Maintenance concepts}

There are some aspects to consider if we want to design an optimal maintenance. The first parameter is the criticity of the task, calculated according to fail consequences, frequency, inactivity time, etc.
With the criticity and the available time to repair the failure, we determine the variable priority of the maintenance task, which should be updated during the program evolution. The priority is very important to determine the most restrictive tasks and also for the grouping system.

Another factor is the possibility of look up the information of the tasks in the corresponding data base.

This system has been designed for offshore wind turbines, so another factor to consider is the shipment used to solve the maintenance task, which cost is really significant in the budgets. Moreover, we have to consider meteorological parameters in order to decide to send the ship or not. This consideration should be analyzed throughout the execution of the program.

The management systems work with at least a complete wind farm. That means a high load of maintenance tasks and parameters that increase even more if all the maintenance types are considered.

The system developed is able to manage all the active maintenance tasks, with the computational load that it supposes. We propose a system implemented using Colored Petri Nets in Labview, with a new characteristic: it can work with any number of maintenance tasks, and grow and decrease the size of the net according to the requirements of the system in every moment.

Commercial management systems have been developed to this kind of system, and are very qualified, but they have some lacks that our system is able to complete, working combined with specific maintenance systems, contributing with an engineer vision of the problem.

Two important aspects for the offshore maintenance are going to be analyzed: the grouping system of the available tasks and the optimization. The grouping system has to consider the aspects we have seen before, and calculate some different options to resolve the tasks. With these options, the optimization system decides which one is the optimal, according to economical and strategic parameters.

The objective of the article is to present an automatic system for the maintenance management, implemented in Labview using a new methodology for self-growing colored Petri nets. 
The maintenance of offshore wind turbines comprises a wide range of tasks, making it a really complicated system. The objective of research in these systems is to improve the efficiency and effectiveness of wind turbines, reducing losses associated with machinery stops and increases the availability of wind farms. [5].

When designing a maintenance management system is necessary to know in detail the parameters of the system and its operation, as well as limiting factors when making improvements in existing systems.

A complete maintenance system must be powered by the types of maintenance that we can see in Table I. This range of methodologies covers all possibilities in diagnostic and predictive failure.

Table I: Maintenance types

\begin{tabular}{|l|l|}
\hline TYPE & DESCRIPTION \\
\hline Preventive & Repairs based on calendar \\
\hline Predictive & $\begin{array}{l}\text { Using condition monitoring to } \\
\text { determine the machine health }\end{array}$ \\
\hline $\begin{array}{l}\text { Corrective } \\
\text { (fault diagnosis) }\end{array}$ & Based on declared failures. \\
\hline $\begin{array}{l}\text { Corrective } \\
\text { (inspections) }\end{array}$ & $\begin{array}{l}\text { Based on failures detected in periodic } \\
\text { revisions. }\end{array}$ \\
\hline Proactive & Analysis of parts, reliability, etc. \\
\hline
\end{tabular}

There are some things to consider if we want to design an optimal maintenance system [add reference Granada]. The first thing to consider is the criticality of the task. This is set based on the consequences of failure, the frequency, the idle time, the available time to repair the fault, etc.

Based on the criticality of the task and the time available for repairs, which vary depending on the type of maintenance input is assigned to each task a priority variable that will be modified over time as the available time decreases . This priority is important for the system to determine the tasks to be performed sooner.

Another important factor for the development of the application is based on the need to view information about the task in the corresponding databases. This information may be the requirements of the task to be performed, availability of equipment and personnel necessary, external factors, etc.

This article refers to the development of systems for offshore wind turbines, so that one of the most important factors is the type of transport used, as well as restrictions on size, that are very important to take into account the high costs posed by these movements, and greatly increase budgets in conventional wind turbines.

One of the requirements when you run a maintenance task is the need for the weather conditions to be appropriate for the task. In terms of offshore, these weather conditions should be added to be also suitable for the boat selected. The maintenance system should keep these restrictions throughout the management process because it is a highly variable parameter.

Another parameter to be analyzed in the high burden of running maintenance tasks with which these systems work as management programs must address the needs of at least an entire wind turbine plant. This involves working with large numbers of orders and parameters, which increases even more if using all entries seen in Table 1. Therefore, the system used must be capable of handling all active orders in conjunction with the computational burden that this entails. This article proposes a system with Colored Petri Nets, whose features, performance and justification for the program is discussed in section 4 .

At present there are plenty of commercial systems for maintenance management, so we will analyze their characteristics to know the restrictions, allowing us to know the areas in which research can be interesting.

These systems have been developed and improved over the years, with the collaboration of maintenance companies, so these programs are very complete. However, these programs are created from a management standpoint, not from the industrial, so there are several areas for improvement.

The characteristics in terms of management and treatment database are excellent, but if you want to incorporate new technologies, they present difficulties. It will be specifically discussed two important aspects for the maintenance offshore, such as the grouping of tasks carried out jointly and optimization.

In both cases, these parameters are determined by the operator's decision about when to run maintenance tasks. As mentioned above, onshore systems are not as restrictive, but for offshore wind turbines become a key point to be developed.

The grouping system should consider the important factors discussed in this paragraph, as the requirements and priorities of tasks, types of boats and weather. Optimization has to decide which of the options for implementation of maintenance orders, the result of the grouping system, is most appropriate in terms of various economic and strategic parameters. When creating an automated management system, the development of grouping and optimization subsystems would be a very important factor in terms of improving existing systems.

The aim of this paper is the creation of an automated maintenance management system, making an improvement to business systems. Therefore, the developed system must meet the requirements of the concepts discussed in this paragraph and shall be implemented in a program that allows the automatic execution of the various subsystems that form the program. 
We can determine, as a conclusion, which the most relevant factors to the development of a maintenance management system are:

- Detailed information about parameters and requirements of the tasks.

- Appropriate use of databases.

- Consider continuous changes in parameters and weather conditions.

- Development of systems of classification and optimization.

- Automate the entire process.

The amount of work orders that are handled with a management system is an important factor for the development of the tool. That is why there is the use of CPN since the structure of the maintenance system is the same for all orders, with differences in transitions, so if you deploy a CPN with colors associated with maintenance orders and properly defined, it can trigger the system to simplify and generalize.

\section{Maintenance system developed}

Now, it's going to be presented the automated system implemented for managing maintenance using Colored Petri Nets. This is a complex system made up of several subsystems, which will be discussed first, the CPN that defines the overall system performance, and then the subsystems, studying the characteristics of each and improvements incorporated in traditional systems.

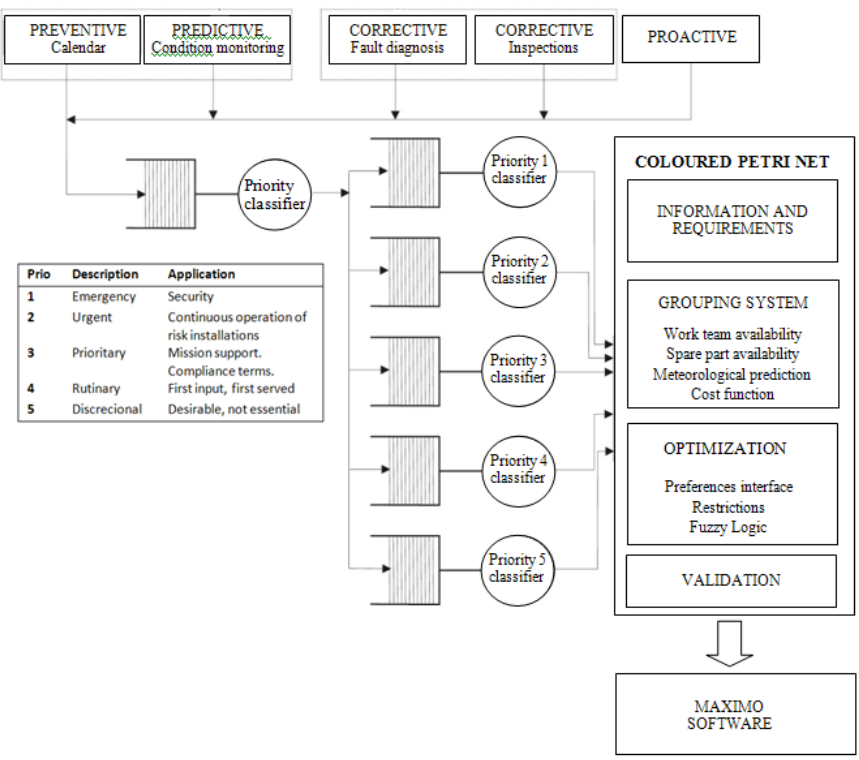

Fig. 1: Maintenance scheme

Figure 1 is proposed as the maintenance scheme. We can see that the entries cover the range of system types used for offshore maintenance systems. Condition monitoring and fault diagnostics modules are used as input for predictive maintenance and corrective type. The other entries are based on research, inspections and calendar.
The first objective of the scheme is to analyze the parameters of the maintenance order to determine, depending on the criticality of the fault and the time available for repair, the value to be assigned as priority. With this factor of priority, the system takes care to separate the orders by priority for grouping different modules. These modules are dynamic, and use the priority and the time available to reorganize inside.

Let's look at the basic structure of the Petri net that will follow each of the tasks created and introduced into the system. The network can be divided into two systems:

- Requirements analysis

- Grouping, optimization and revision

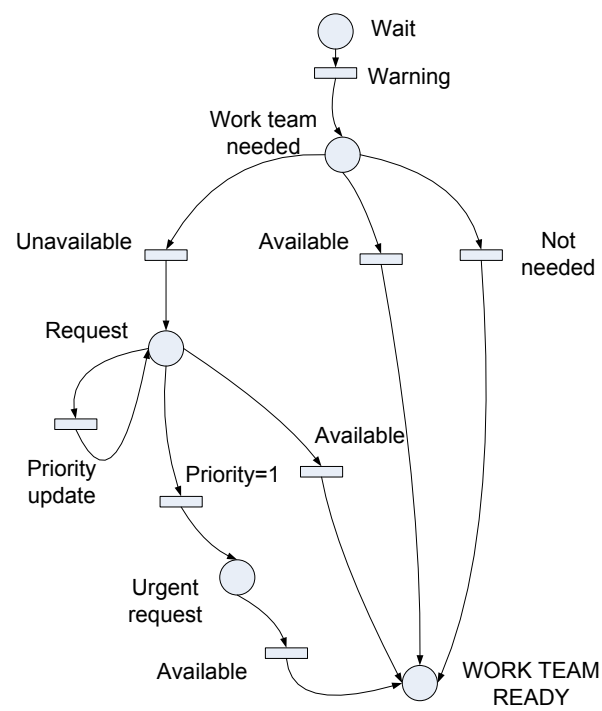

Fig. 2: Petri Net for the Work Team

The first subsystem (Figures 2 and 3) simultaneously performs a data query in the order information about the needing of material and qualified personal, determining first whether it is necessary to any of these parameters and at a later stage the characteristics of the requirements (hours, number, etc.).

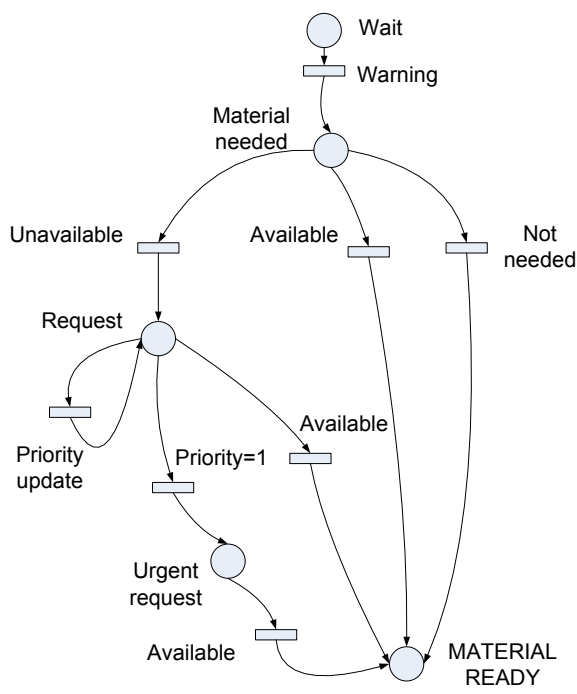

Fig. 3: Petri Net for the Material 
During the process the task priority is constantly being updated, depending on the time available. If this is the case of reaching emergency levels of performance, urgent request is made of the requirements that have not been fulfilled. Once the requirements for the preparation of the order are met, the order stays in a waiting stage, pending the weather conditions to be favorable for proceeding to the second subsystem.

This second stage of the Petri net (Figure 4) takes care of the different groups according to the active tasks and calculates, using different methodologies, the optimal solution based on the parameters set by the system. Subsequently, validation is required by the operator, allowing recalculation of the options if it is considered that the optimal option is not appropriate. Throughout the whole process, the system keeps updating priorities and reviewing the weather conditions.

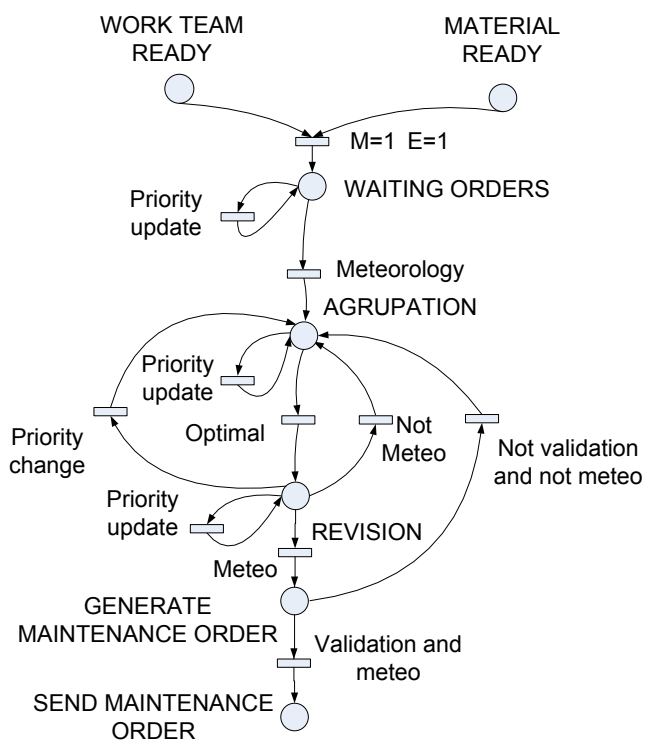

Fig. 4: Petri Net for the Optimization and Revision Subsystem

The grouping phase must consider the possibilities of transport to the turbine plant (type of ship, size, capacity, ability to use helicopter, etc.) must be balanced against the costs associated with carry out the duties and shipping of the boat. With these parameters, the system calculates the possible options for grouping tasks that will move to the optimization subsystem.

For the optimization, there have been proposed several different methods for calculating the optimal solution. It is important to have a range of options, because the accuracy of the information with which you work depends largely on the company or the type of machine with which it works, and since the methodologies are based on different parameters solutions may not be equal.

The proposed methodologies are:

- Constraint Based

- Preference interface based $(\mathrm{CPOH})$

- Fuzzy Logic
The last step of the net is going through the review and acceptance of the operator in charge, in command of the maintenance system and complying with the parameters set by the company. To meet this objective, the system should inform the user through Maximo of the different grouping solutions and their parameters and the option considered optimal. Apart from the validation of the operator, it is necessary that the weather conditions continue being satisfied, since it is a parameter subject to change.

Once the user has selected the best option, all other orders that have not been executed should return to standby to allow for future clustering.

Throughout the process, the system should remain the priority update of each task, as this parameter varies with time and determines the urgency of repair, which may modify the grouped options.

\section{Self-growing Colored Petri Nets}

Here, we are going to present the methodology used for the Colored Petri Net implementation in Labview. We have analyzed previously the characteristics of the maintenance system that justify the requirement of a new kind of Petri Net implementation.

The methodology consists in a basic CPN structure, which is duplicated every time a new maintenance order is created, without affecting the normal operation of the system. That means that the system is dynamically working and the CPN grows or decreases the size without stopping the operation. We use the name of the order as the color for the net.

The following parameters are used: origin place, transition number, initial marking, destination place and final marking. Origin and destination place refer to the CPN place where the mark starts and ends with the transition firing. The initial marking is the color mark necessary in the origin to fire the transition, and the final marking is the color placed in the destination place.

Table II: RdPC structure in Labview

\begin{tabular}{|c|c|c|c|c|}
\hline $\begin{array}{c}\text { Origin } \\
\text { Place }\end{array}$ & $\begin{array}{c}\text { Transition } \\
\text { number }\end{array}$ & $\begin{array}{c}\text { Initial } \\
\text { Marking }\end{array}$ & $\begin{array}{c}\text { Destination } \\
\text { Place }\end{array}$ & $\begin{array}{c}\text { Final } \\
\text { Marking }\end{array}$ \\
\hline
\end{tabular}

With this structure it's not difficult to add or substract more CPN, but the challenge is to make all the system generic and related to the index of the order. It's not about making the structure grown and reinitialize the system, but work with a structure dynamically changing.

For the correct operation of the tool should be available also the vectors of inputs and transitions, which like the previous structure will resize depending on the active work orders. 
The system uses a Petri net to assess the conditions of the various tasks. The various types of maintenance feed the system, including in each case the relevant information to the task. This information is entered into the Excel file about new maintenance order, including an identifier of the task.

Thus, the system implemented in Labview is capable of generating a new work order completely independent of the above, changing the initial structure of the Petri net implemented, by the use of the self-growing colored Petri Net developed and described previously.

The operation is as follows: we define a Petri Net structure base, which will be discussed in detail below, with transitions and system inputs. From this point, each new work order repeats the previous structure, while maintaining an identifying code of the task.

The system must be able to run with the number of orders that are active at the time, which can be very small or very large depending on the time. This will mean that the CPN does not have a certain size, but should be a dynamic structure.

Thus, we are working with several duplicate Petri nets but independent. This is a great advantage over traditional methods of Petri nets, can generate as many orders as necessary maintenance, because the whole system is automated and operates on the basis of the identifier of the order.

Dimensiones del sistema con órdenes existentes

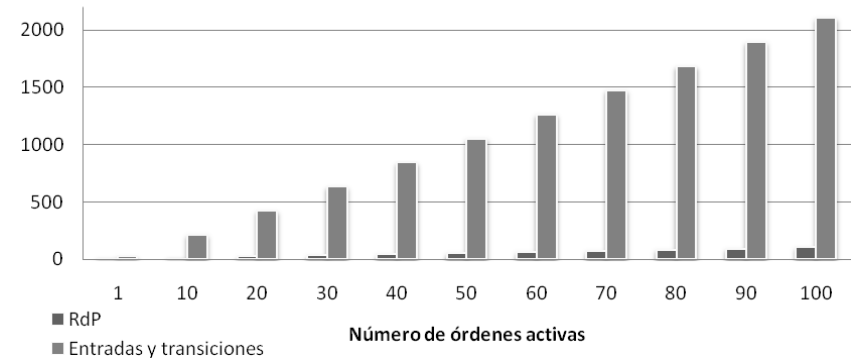

Fig. 5: Dimensiones RdPC

Figure 5 shows how the system size increases depending on the number of active orders. As can be seen, depending on the increase in this number, the number of duplicates of the original network increases, and also the number of entries and transitions this image tries to show the difference between working with 20 orders and less than 500 entries, or 10 orders and more than 2000 entries.

The system is designed is fully prepared to raise the entire structure each time it receives a work order, maintaining the previously active orders in the state in which they were, dynamically growing.

Using the same methodology, once the order has been executed and is drawn from the maintenance system, the tool is able to erase its structure, updating the corresponding parameters, without affecting the normal operation of the system.

The system is greatly based in the communication between Labview and Excel. The system is implemented in Labview, and works in it, but the CPN structure and the transitions and inputs vectors are defined in Excel, so the libraries to the communication have been highly developed.

This connection between the programs is really useful for the query about the requirements of the orders, due to the ability of the software MAXIMO to send the information in Excel format.

\section{Conclusions}

In this paper, we present a complete automatic maintenance system for offshore wind turbines, working with the recognized software Maximo, and a new methodology that allows the system to work with all the active maintenance tasks in the moment, by a selfgrowing architecture and performance.

The methodology has been developed specifically for the offshore maintenance system, but the modularity of the performance makes possible to adapt it to so many other systems, just adjusting the structure of the basic Colored Petri Net.

The libraries developed facilitate the communication between the programs, an indispensable task for the present system.

This new methodology is going to be implemented in an offshore wind turbine project, for the maintenance system. This way, the methodology tries to reduce maintenance costs by using a dynamic system with optimization, and also tries to help the offshore industry to enhance their development.

\section{References}

[1] García, E., Morant, F., Correcher, A., Quiles E. "Seguimiento de Estado, Diagnóstico de Fallos y Mantenimiento Industrial: una visión integrada." Proceedings of the Novena Semana Tecnológica. 19-23 October 2009. La Habana.Cuba.

[2] Pérez M., García E., Morant F., Correcher A. and Quiles E. "Optimal Maintenance system for offshore wind turbines". International Conference on Renewable Energies and Power Quality (ICREPQ'10).Granada (Spain), 23th to 25th March, 2010

[3] M. García, M.A. Sanz and J. Del Pico, "Aplicación de técnicas de inteligencia artificial en el mantenimiento predictivo de aerogeneradores", in IV Jornada de Fiabilidad: Confiabilidad, Las Palmas de G.C., September 2002.

[4] L.W.M.M. Rademakers, H. Braam, T.S. Obdam, P. Frohböse, N. Kruse, "Assessment and optimization of operation and maintenance of offshore wind turbines", Proceedings of the European wind energy conference, 16-19 June, Madrid, Spain, EWEA (2003) 6 pp. 